\title{
Les virus de l'immunodéficience sont-ils eux-mêmes déficients
}

Les virus de l'immunodéficience humaine (HIV l et 2) aussi bien que simienne (SIV) ne sont guère pathogènes chez l'animal d'expérience $\left(\mathrm{m} / \mathrm{s} n^{\circ} 10\right.$, vol. 4, p. 658), si bien que l'une des seules maladies animales ressemblant vraiment au SIDA est l'affection du chat causée par une souche du virus de la leucémie féline FeLV. Le génome de ce virus est « défectif », c'est-à-dire qu'il est incapable, par lui-même, de se répliquer, ayant besoin pour ce faire de l'apport des fonctions d'un virus auxiliaire dit helper. Ce virus de l'immunodéficience féline a, notamment, un gène d'enveloppe (env) anormal qui semble porter le pouvoir pathogène. Plus récemment, une équipe américaine [1] et une équipe canadienne de l'université de Montréal, dirigée par P. Jolicœur [2], ont caractérisé des souches de virus de la leucémie murine susceptibles d'entraîner un syndrome d'immunodéficience sévère chez la souris. Les virus d'une préparation de ganglions de souris immunodéficiente ont été passés sur culture de cellules, puis utilisés pour infecter d'autres souris chez qui ils ont provoqué un syndrome ressemblant au SIDA après un délai de huit à 12 semaines. La préparation de virus infectants comporte un mélange de génomes viraux entiers et de génomes défectifs qui ont été clonés séparément. La pathogénicité semblait plus étroitement associée à la richesse des extraits en formes défectives qu'en formes intactes. Les génomes viraux clonés ont été utilisés pour diriger la formation en culture cellulaire des particules virales (avec l'aide d'un virus helper). Seules les particules virales à génome défectif se sont révélées pathogènes. Le génome viral normal a une taille de $8,8 \mathrm{~kb}$,- alors que le génome défectif délétions des gènes env (enveloppe) et pol (polymérase, transcriptase inverse). Il existe, dans le gène gag (codant pour une protéine de la capside virale,) une séquence polypeptidique de $12 \mathrm{kDa}$ qui semble caractéristique des formes pathogènes et pourrait être responsable de la pathogénicité, par un mécanisme encore inconnu.

Peut-on, à partir de cet exemple, élargir le concept de l'intervention de virus (défectifs ou déficients) dans des maladies? Certainement, oui. D'abord parce que de nombreux rétrovirus oncogéniques sont, de fait, défectifs. Puis, parce que dans le cas même des virus de type HIV, rien n'indique à coup sûr que les formes compétentes pour la réplication soient celles qui sont responsables de la symptomatologie du SIDA. Ces incertitudes amènent même P.H. Duesberg, un distingué académicien des sciences des Etats-Unis, virologue sérieux, à contester la responsabilité, au moins exclusive, des virus HIV dans le SIDA [3]. Sans aller jusque-là, on sait que la dérive génétique des virus HIV est telle que coexistent toujours, chez les malades, de très nombreux types de virus, les uns réplicatifs, les autres non (S. Wain Hobson, Institut Pasteur de Paris, communication personnelle). Un schéma dans lequel l'infection se ferait par des virus réplicatifs, dont pourraient dériver des mutants multiples, certains défectifs et pathogènes, est donc parfaitement plausible. Il pourrait expliquer le très long temps de latence entre l'infection et la maladie... et peut-être aussi la faible efficacité, au moins à terme, des drogues antivirales s'opposant à la réplication, telle l'AZT [4].
1. Weiss RA. Defective viruses to blame? Nature 1989; $338: 458-60$.

2. Aziz DC, Hannaz, Jolicœur P. Severe immunodeficiency disease induced by a defective murine leukaemia virus. Nature 1989 ; 338 : $505-8$.

3. Duesberg PH. Human immunodeficiency virus and acquired immunodeficiency syndrome: correlation but not causation. Proc Natl Acad Sci USA 1989; 86 : 755-64.

4. Larder BA, Darby G, Richman DD. HIV with reduced sensitivity to zidovudine (AZT) isolated during prolonged therapy. Science $1989 ; 243: 1731-4$

\section{口D BRÈVES}

- L'antigène de la leucémie aiguë lymphoblastique commune (CALLA) est l'enképhalinase. L'antigène CALLA (common acute lymphoblastic leukaemia antigen) est un marqueur classique de la membrane des lymphoblastes leucémiques humains. Son gène a récemment été cloné par des équipes américaines de Boston (MA) et Dallas (TX) [1]. La séquence a permis de montrer que la protéine CALLA était identique à une endopeptidase neutre qui inactive un grand nombre de neuropeptides et qui est connue sous le nom d'enképhalinase. De fait, des lymphocytes transfectés par un vecteur d'expression dirigeant la synthèse de CALLA acquièrent également une activité enképhalinasique. Cette étonnante constatation rajoute une pièce au dossier des relations entre le système immunitaire et le système nerveux central $\left(m / s n^{\circ} 7\right.$, vol.2, p. 401).

[1. Shipp MA, et al. Proc Natl Acad A.K. Sci USA $1989 ; 86$ : 297-301.] 
L'accumulation de mutations somatiques du génome mitochondrial explique t-elle certains des déficits fonctionnels du vieillissement ? Le génome mitochondrial humain est incroyablement compact ; pratiquement chacun de ses 16596 nucléotides est «signifiant», ce qui veut dire que toute mutation de ce génome pourra avoir des conséquences fonctionnelles... au moins pour la mitochondrie qui la porte. De nombreuses maladies génétiques associées à des anomalies diverses de l'ADN mitochondrial ont été décrites ces dernières années, et il semble que le rythme de ces descriptions s'accélère actuellement, l'attention de la communauté scientifique étant fortement attirée sur cette nouvelle pathologie $\left(m / s n^{\circ} 2\right.$, vol. $\left.5, p 123\right)$. Une équipe australienne (Melbourne et Victoria) vient récemment de démontrer que les mitochondries de muscles squelettiques de sujets âgés ont, en moyenne, une réduction de leur capacité respiratoire, ainsi qu'une diminution progressive de l'activité d'enzymes sous contrôle du génome mitochondrial, alors que l'activité d'une enzyme mitochondriale codée par l'ADN nucléaire reste stable [1]. L'exercice musculaire soutenu... ainsi que l'activité cérébrale puisent principalement leur énergie dans les phosphorylations oxydatives mitochondriales, si bien que toute réduction de ce côté amoindrira les performances de ces deux tissus... ce qui est bien constaté dans le vieillissement. A partir de ces données, une équipe autralo-japonaise (Victoria et Nagoya) fait l'hypothèse que les mutations somatiques du génome mitochondrial sont constantes tout au long de la vie et, à partir d'un certain âge, aboutissent à de sévères réductions de performance, variables d'une cellule et d'un tissu à l'autre du fait de l'« hétéroplasmie », c'est-àdire de la coexistence en proportions variables dans une même cellule de mitochondries normales et de mitochondries à génome modifié [2]. Si cette hypothèse est juste, elle pourrait déboucher sur des tentatives théra$m / s n^{\circ} 6$ vol. 5, mai 89 peutiques utilisant diverses substances (des transporteurs d'électrons) susceptibles de pallier certaines des anomalies mitochondriales.

[1. Trounce I, et al. Lancet $1989 ; 1$ : 637-9.]

[2. Linnace AW, et al. Lancet 1989 ; $1: 642-5$.]

Les molécules de classe II du complexe majeur d'histocompatibilité et la protéine d'adhérence cellulaire ICAM-1 suffisent à reconstituer une fonction de présentation de l'antigène. Les antigènes sont normalement présentés par une molécule du complexe majeur d'histocompatibilité $(\mathrm{CMH})$ au récepteur pour l'antigène des lymphocytes $T$. Outre cette interaction $\mathrm{CMH} /$ récepteur $\mathrm{T}$, d'autres interactions entre molécules d'adhérence cellulaire [1] semblent indispensables pour stabiliser le contact entre la cellule présentatrice et le lymphocyte $\mathrm{T}$ : les molécules CD2, CD4, CD8 et LFA-1 sont présentées à la surface de cellules T cytotoxiques ou auxiliaires, les molécules LFA-3 (ligand de CD2) et ICAM-l (ligand de LFA-1) étant exprimées à la surface des cellules présentatrices, par exemple les monocytes. Une équipe londonienne dirigée par D. Wilkinson vient de démontrer que des fibroblastes transfectés par des gènes codant pour des molécules de classe II du CMH et pour la molécule ICAM-1 devenaient de bonnes cibles pour des lymphocytes $T$ cytotoxiques spécifiques qui étaient activés par contact avec ces fibroblastes transfectés. Il se pourrait que ICAM-1 intervînt non seulement dans la stabilisation du contact entre la cellule cible et le lymphocyte $T$, mais aussi dans la «transduction» du signal d'activation, comme cela a déjà été évoqué aussi bien pour le couple ICAM-1/LFA-1 que pour LFA-3/CD2 (m/s suppl. au $n^{\circ} 1$, vol. 5, p. 28).

[1. Fisher A, et al. médecine/sciences $1987 ; 3: 334-42$.

[2. Altmann DM, et al. Nature 1989 ; $338: 512-4$.
Le traitement médical des calculs biliaires à cholestérol. Les traitements visant à éliminer les calculs biliaires sans avoir recours à la chirurgie ne sont pas encore entrés dans la pratique courante. L'emploi des ondes de choc $\left(\mathrm{m} / \mathrm{s} n^{\circ} 6\right.$, vol. 2 , p. 343) n'a que très peu de cas à son actif. La dissolution médicamenteuse par les acides chénodésoxycholique et ursodésoxycholique réclame un à deux ans. En 1985, une équipe de la Mayo Clinic proposait l'emploi du méthyl-tert-butyléther (MTBE), similaire à l'éther éthylique, mais moins dangereux car moins volatil $\left(\mathrm{m} / \mathrm{s} n^{\circ} 3\right.$, vol. 1, p. 161). Les résultats ne portaient que sur trois malades et réclamaient confirmation. Ceux que vient de publier la même équipe [1] englobent 75 malades. Rappelons d'abord que le MTBE, très actif in vitro pour dissoudre les calculs de cholestérol, est instillé dans la vésicule biliaire par un cathéter transhépatique percutané, sous contrôle fluoroscopique, puisque les calculs de cholestérol sont invisibles aux rayons $\mathrm{X}$. Le MTBE est instillé et aspiré quatre à six fois par minute, en moyenne pendant cinq heures, un à trois jours. Les effets secondaires douleurs, fuites de bile ou du liquide injecté - n'ont jamais empêché la bonne marche du traitement. Sur les 75 malades, 72 virent leurs calculs dissous entièrement ou à $95 \%$. Une nette amélioration du pronostic a été observée avec les progrès de la technique : cinq des six premiers sujets traités, mais un seul des 69 suivants, ont dû subir ultérieurement une cholécystectomie, sur une période de suivi de six à 42 mois. Les résultats de cette méthode, qui a l'immense avantage de supprimer les calculs en un à trois jours, sont donc encourageants, si l'indication est correctement posée. La plus grande difficulté reste d'être sûr que le ou les calculs sont formés uniquement, ou essentiellement, de cholestérol, condition indispensable à l'efficacité.

[1. Thistle JL, et al. N Engl J Med $1989 ; 320$ : 636-9.] 